\title{
Tumor teratoide rabdoide atípico en un adulto joven
}

\author{
Atypical teratoid rhabdoid tumor in an adult patient
}

Carlos Andrés Quintero', Christian Castro², Fernando Hakim³, Nicolás Useche ${ }^{4}$, Marcela Mejía ${ }^{5}$, Adriana Flórez ${ }^{5}$,
Sonia Bermúdez ${ }^{4}$, Fernando Velandia ${ }^{5}$, Alfredo García ${ }^{5}$, León Darío Ortiz ${ }^{6}$, Andrés Felipe Cardona ${ }^{7,8}$

IInstituto de Neurociencias, Universidad El Bosque, Hospital Universitario Fundación Santa Fe de Bogotá (Bogotá, Colombia). 2Facultad de Medicina, Universidad de los Andes, Fundación Santa Fe de Bogotá (Bogotá, Colombia).

${ }^{3}$ Departamento de Neurocirugía, Fundación Santa Fe de Bogotá (Bogotá, Colombia).

${ }^{4}$ Departamento de Imágenes Diagnósticas, Sección Neurorradiología, Fundación Santa Fe de Bogotá (Bogotá, Colombia).

${ }^{5}$ Departamento de Patología y Laboratorio Clínico, Fundación Santa Fe de Bogotá (Bogotá, Colombia).

${ }^{6}$ Grupo Neurooncología, Departamento de Oncología Clínica, Instituto de Cancerología, Clínica Las Américas (Medellín, Colombia).

${ }^{7}$ Grupo Oncología Clínica y Traslacional, Instituto de Oncología, Fundación Santa Fe de Bogotá (Bogotá, Colombia).

${ }^{8}$ Fundación para la Investigación Clínica y Molecular Aplicada del Cáncer (FICMAC); investigador asociado ONCOLGroup.

\section{Resumen}

Se presenta el caso de un hombre de 28 años con pérdida progresiva de la agudeza visual y cefalea asociada a polidipsia, poliuria e hipersomnia. La resonancia magnética cerebral mostró una masa heterogénea localizada a nivel selar/supraselar con realce intenso después de la administración del contraste. Se realizó una resección parcial con patología que evidenció una lesión tumoral de apariencia rabdoide con pérdida de la expresión del INI-1. Estos hallazgos fueron compatibles con un tumor teratoide/rabdoide atípico tratado con teleterapia y el esquema de quimioterapia ICE con adecuada respuesta. Se discute el resultado después de cinco meses de tratamiento en espera de la administración de quimioterapia con intensidad de dosis más rescate con progenitores obtenidos de sangre periférica. También se presenta una revisión de los datos referentes al manejo de esta neoplasia, al igual que de los mecanismos moleculares implicados en su fisiopatología.

Palabras clave: Tumor teratoide/rabdoide atípico, adulto, quimioterapia, radioterapia, patología molecular, trasplante.

\begin{abstract}
The case of a 28-year-old man is presented; he was suffering from a progressive loss of visual acuity and headache associated with polydipsia, polyuria and hypersomnia. Brain magnetic resonance spectroscopy revealed a heterogeneous mass located in the sellar/suprasellar areas having intense enhancement after contrast. A partial resection was made, pathology revealing a rhabdoid tumoral lesion accompanied by loss of INI-1 expression. Such findings were compatible with an atypical teratoid/ rhabdoid tumour which was then treated with teletherapy and ICE chemotherapy which led to a suitable response. The result following five months' treatment pending the administration of dose-intensity chemotherapy plus peripheral-blood progenitor cells (PBPC) as hematopoietic rescue is discussed. A review of the pertinent data regarding managing this neoplasia is also presented, along with the molecular mechanisms implicated in its physiopathology.
\end{abstract}

Key words: Atypical teratoid/rhabdoid tumor, chemotherapy, radiation, molecular pathology, transplant.

\section{Introducción}

El tumor teratoide rabdoide atípico (AT/RT, por su sigla en inglés) es una entidad poco frecuente que representa entre el $1 \%$ y $2 \%$ de los tumores cerebrales en la población pediátrical-3; esta neoplasia tiene un alto potencial de malignidad, por lo que ha sido clasificada dentro del grupo de neoplasias grado IV, según la clasificación de la Organización Mundial de la Salud
$(\mathrm{OMS})^{4}$. El AT/RT tiene un pico de incidencia entre los niños menores de tres años y suele ser dominante en el sexo masculino. De forma regular, la mediana de supervivencia global (SG) no supera los 24 meses $^{5-7}$ y solo se han reportado 34 pacientes adultos que presentan una edad promedio de 30 años $^{8}$.

Su diagnóstico representa un reto debido a la heterogeneidad celular que incluye componentes 
rabdoides, neuroepiteliales primitivos, epiteliales y mesenquimales ${ }^{3}$. Por lo anterior, es importante considerar como parte del diagnóstico diferencial a los meduloblastomas y a los tumores neuroectodérmicos primitivos (PNET) $)^{4}$.

La inmunohistoquímica es una herramienta diagnóstica valiosa, debido a que permite excluir ciertas patologías que guardan similitud fenotípica. El tumor suele ser positivo para el antígeno epitelial de membrana (EMA), vimentina, citoqueratinas, proteína ácida fibrilar glial (focal), desmina, S-100, actina de músculo liso, y, ocasionalmente, se encuentra expresión de sinaptofisina ${ }^{9}$. Sin embargo, el marcador más relevante es la detección de la mutación (22q11.23) relacionada con la alteración citogenética 9:22x, que induce pérdida tanto de la función del INI-1 como de su expresión proteica por inmunohistoquímica ${ }^{10}$.

El tratamiento del AT/RT en adultos se ha extrapolado de la población pediátrica, incluyendo una visión multimodal que inicia con la resección quirúrgica máxima (teniendo como meta una mínima volumetría posoperatoria), seguida de la adyuvancia con radioterapia y quimioterapia de múltiples agentes, considerando el uso de la temozolamida, ifosfamida, carboplatino y etopósido (ICE) ${ }^{8}$.

Debido al advenimiento de la genómica tumoral, se ha reconocido una diversidad de vías implicadas en el desarrollo del AT/RT (p16 INK4a, E2Fs y ciclina D1, Akt/Mtor, Aurora A), hallazgos que han permitido estudiar nuevos blancos terapéuticos que podrían optimizar la respuesta minimizando la toxicidad. A continuación, se presenta el caso de un adulto con el diagnóstico de un AT/RT de la región selar y supraselar, con la intención de explorar el comportamiento de la patología para proponer diversas hipótesis sobre el tratamiento. Vale la pena mencionar que este caso es el octavo reportado en la literatura médica para esta localización y el primero en un hombre.

\section{Presentación del caso}

Se trata de un hombre de 28 años sin antecedentes personales de importancia exceptuando la presencia de un meningioma en la abuela materna, quien consultó por un cuadro clínico que inició en mayo del 2012 con la pérdida progresiva de la agudeza visual por el ojo izquierdo, asociada a cefalea tipo peso bifrontal de moderada a severa intensidad. Dos semanas antes de la consulta inicial presentó polidipsia, poliuria e hipersomnia progresiva. En la valoración inicial, el paciente se encontraba estable desde el punto de vista hemodinámico, al examen neurológico estaba somnoliento y su evaluación de la esfera mental fue normal. No obstante, se documentó amaurosis izquierda y un defecto pupilar aferente, así como borramiento de los bordes papilares al fondo de ojo. Los demás parámetros del examen neurológico fueron normales.

Al principio fue evaluado extrainstitucionalmente por oftalmología, que descartó cualquier patología ocular, motivo por el que se remitió a evaluación neurológica. Se llevó a cabo una resonancia magnética cerebral contrastada que demostró una masa extraaxial heterogénea y lobulada de $50 \times 60 \times 55$ mm, localizada a nivel selar y supraselar, con efecto compresivo sobre los lóbulos frontales y el tercer ventrículo. La lesión tenía un realce intenso con el medio de contraste y heterogeneidad que sugirió como primera posibilidad un meningioma o adenoma invasor (figura 1).

Fue remitido al servicio de neurocirugía (Fundación Santa Fe de Bogotá), donde se realizó una resección parcial por medio de un abordaje subfrontal bilateral. La patología encontró una lesión tumoral maligna de aspecto heterogéneo, con áreas celulares de
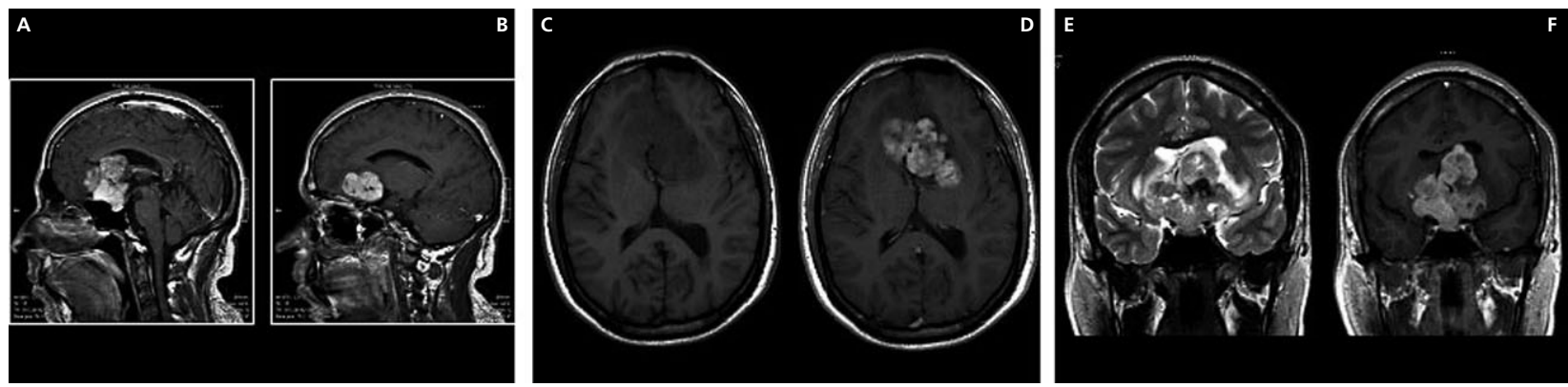

Figura 1. A y B. Cortes sagitales T1 con contraste que muestran extensión selar y supraselar de la masa con compresión sobre el tercer ventrículo y la lámina terminal con realce intenso y ligeramente heterogéneo. C. Corte axial T1 simple. D. Corte axial T1 con contraste mostrando compresión sobre los lóbulos frontales. E. Corte T2 coronal que muestra heterogeneidad de la señal de la masa sin calcificaciones, hemorragia o zonas quísticas. F. Corte T1 coronal con contraste. 


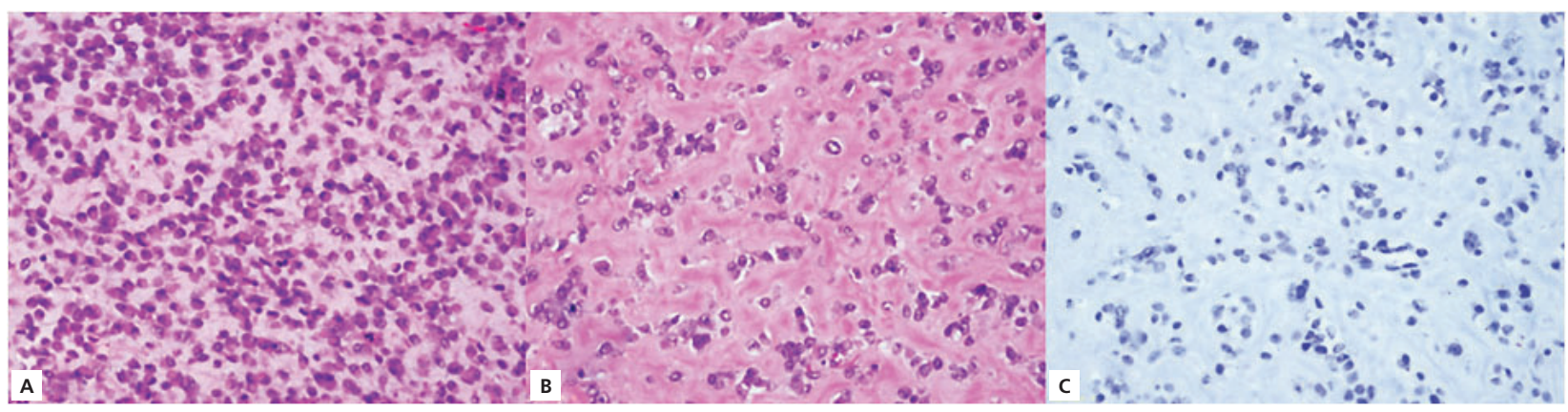

Figura 2. A. En el estudio histopatológico, se reconoce un tumor maligno de apariencia heterogénea, con áreas hipercelulares compuestas por células de núcleo excéntrico con citoplasma eosinófilo de apariencia rabdoide. B. Otras áreas con apariencia condroide. C. El estudio de inmunohistoquímica muestra la pérdida de la expresión del INI-1. (A y B. Hematoxilina eosina. Magnificación original 40X. C. Inmunohistoquímica para INI-1. Magnificación original 40X).

apariencia rabdoide (figura $2 \mathrm{~A}$ ) y otras con matriz condroide (figura 2B), frecuentes mitosis y mínimo pleomorfismo celular. Los estudios de inmunohistoquímica demostraron positividad focal para EMA, vimentina y CD34, así como negatividad para S100, PFAG y sinaptofisina. Como hallazgo singular, se evidenció pérdida de la expresión de INI-1 (figura 2C). Los hallazgos histológicos fueron de una neoplasia maligna con hallazgos rabdoides, que planteó como diagnósticos diferenciales el AT/RT, un meningioma rabdoide, un carcinoma mioepitelial, un linfoma o cordoma; no obstante, los hallazgos de inmunohistoquímica favorecieron el primer diagnóstico. Dado lo inusual de la lesión, el caso fue enviado a evaluación por la Dra. Rebecca D. Folkerth del Departamento de Neuropatología del Brigham's and Women's Hospital (Boston, MA, EE. UU.), quien apoyó el diagnóstico de AT/RT.

Debido a la complejidad histológica, se consideró inicialmente la presencia de un linfoma plasmablástico,

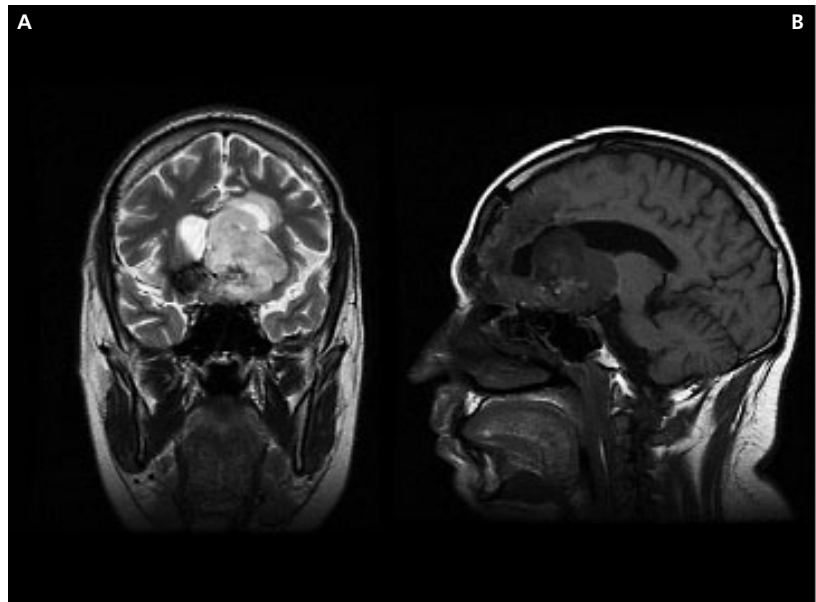

por lo cual se administró metrotexate (altas dosis) con toxicidad hepática autolimitada. Después de confirmar el diagnóstico de AT/RT, se administró teleterapia temprana (6.000 cGy/200 cGy), seguida de poliquimioterapia con el esquema ICE (ifosfamida $2.000 \mathrm{mg} / \mathrm{m}^{2}$ días 1 a 3, carboplatino $400 \mathrm{mg} / \mathrm{m}^{2}$ días 2 y 3, y etopósido $150 \mathrm{mg} / \mathrm{m}^{2}$ días 1 a 3, cada 21 días), con adecuada respuesta en el control imaginológico realizado a los cuatro meses, que mostró una disminución volumétrica mayor al 50\% (figura 3). Vale la pena mencionar que, después del primer ciclo, se llevó a cabo una recolección de progenitores obtenidos en sangre periférica con el objeto de realizar intensificación de dosis más trasplante autólogo.

En el momento, el paciente completó cinco meses de tratamiento con mejoría parcial del déficit neurológico inicial, persistiendo un recorte campimétrico en infraversión. Se documentó hipogonadismo central, que se encuentra en manejo endocrinológico con adecuado control. La última resonancia magnética cerebral con

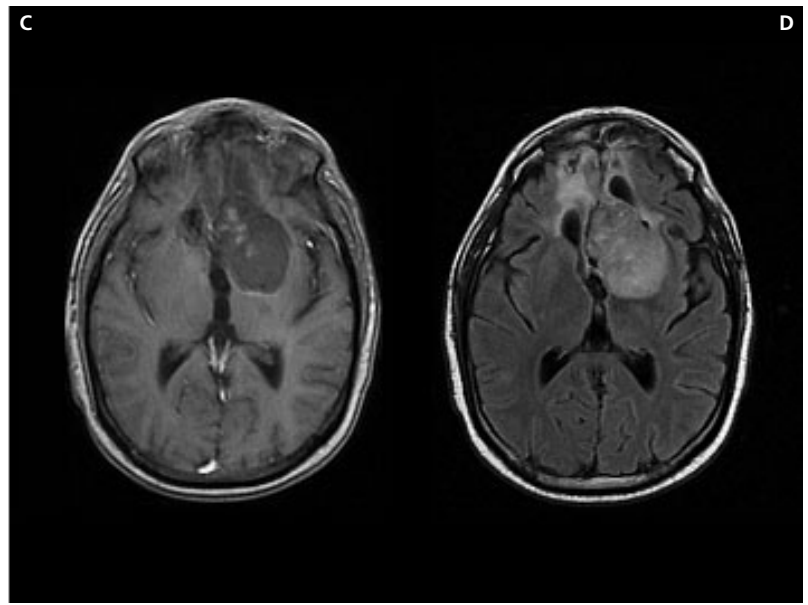

Figura 3. A. Secuencia T1 con contraste demuestra disminución en el realce, tamaño y efecto compresivo de la masa sobre los lóbulos frontales y el sistema ventricular especialmente en el lado derecho. B. Secuencia FLAIR axial que demuestra cambios posquirúrgicos. C y D. Secuencia T2 coronal y T1 sagital (respectivamente), que muestran disminución del tamaño, del efecto de masa y un pequeño foco de sangrado en el lado derecho de la lesión residual. 

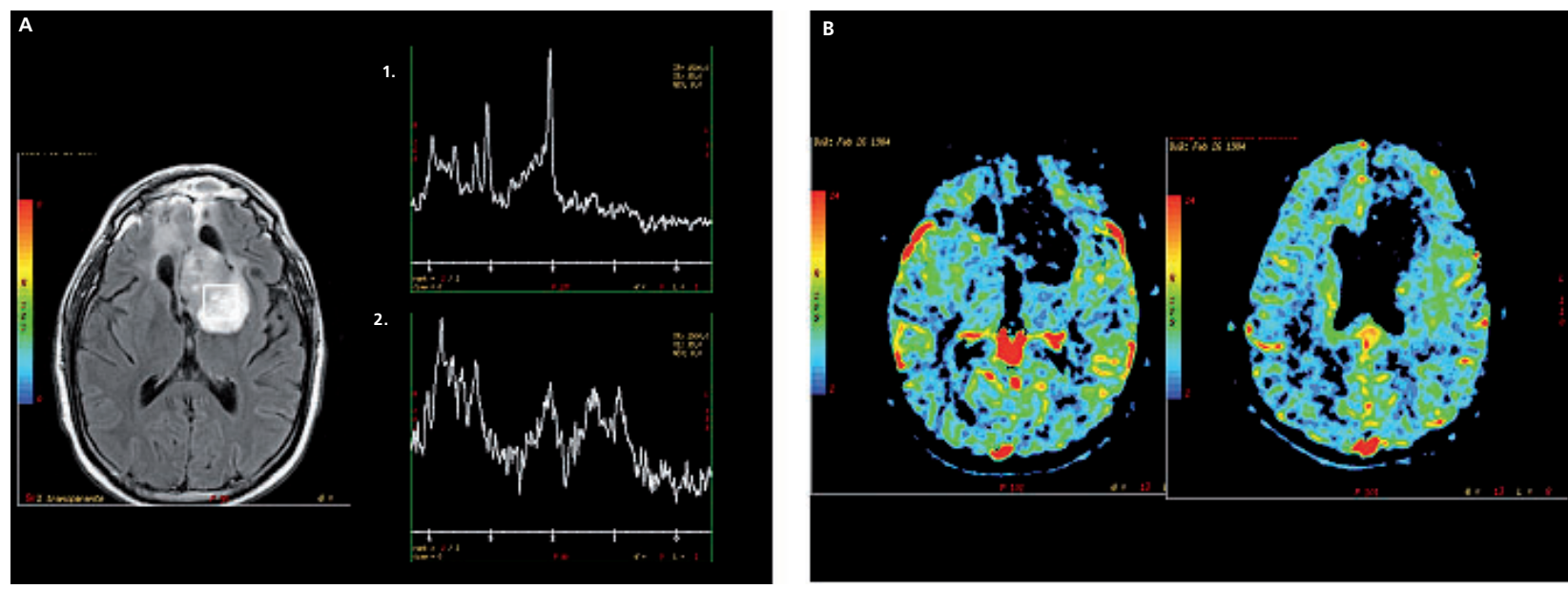

Figura 4. A. Espectroscopia con técnica univoxel que está localizada sobre la masa. A1: espectro de metabolitos en el parénquima normal. A2: espectro de metabolitos en la masa, demostrando dupleta de lactato, disminución del NAA, estabilidad de creatina, elevación leve de la colina y pico en 4 ppm de significado indeterminado. B. Secuencia de perfusión de realce dinámico con contraste, que no demuestra aumento en la perfusión.

técnicas avanzadas indicó enfermedad estable, sin evidenciar áreas con aumento de la permeabilidad capilar que sugieran progresión tumoral. La espectroscopia reveló una disminución del pico de $\mathrm{N}$-acetilaspartato (NAA), con aumento leve del pico de colina (Cho) y presencia de un pico de lactato (figura 4).

\section{Discusión}

La primera descripción de un AT/RT se realizó en 1978, cuando Beckwith y Palmer acuñaron el término de "tumor rabdoide" para describir una variante histológica del tumor de Wilms que se presentaba en pacientes pediátricos con mal pronóstico'. Sin embargo, la primera descripción de un tumor rabdoide primario del sistema nervioso central se hizo en $1985^{2}$. Una década después, Rorke y colaboradores lo llamaron tumor teratoide/ rabdoide atípico debido a la combinación dispar de sus componentes patológicos ${ }^{3}$.

Su presentación clínica varía dependiendo de la localización, se caracteriza por la presentación de letargia, cefalea, emesis, alteración en pares craneales y hemiplejía. Tanto en pacientes pediátricos como en adultos, la localización más frecuente es a nivel supratentorial, teniendo mayor tropismo por el lóbulo frontal. A nivel infratentorial, se han descrito casos en los hemisferios cerebelosos, y menos común es la ubicación en el ángulo pontocerebeloso, en la glándula pineal y en el tallo cerebral ${ }^{9}$. Como se mencionó, nuestro caso es el octavo documentado en el área selar $(7)^{10-13}$ y supraselar (1) ${ }^{14}$.

Desde el punto de vista imaginológico, no existen hallazgos distintivos para el AT/RT, no obstante en las secuencias con información T1 se menciona la presencia de áreas hiperintensas asociadas a sangrado intratumoral, como lo muestran las imágenes del paciente descrito. Tras la administración del medio de contraste, el tumor presenta un realce variable y en las imágenes con información T2 suele ser heterogéneo ${ }^{15}$

La morfología documenta células grandes, poligonales con núcleos excéntricos vesiculares, nucléolos prominentes e inclusiones intracitoplasmáticas eosinofílicas que corresponden con los agregados de filamentos intermedios característicos de las células rabdoides ${ }^{9}$. Genotípicamente los pacientes con AT/RT presentan pérdida de un alelo del cromosoma 22 (monosomía 22) o una deleción/traslocación relacionadas con la banda 22q11.2, supresión que abarca el locus del gen INI-1 (SMARCB1, hSNF5, BAF47) localizado en 22q11.23 y que consta de nueve exones. La proteína expresada por el INI-1 hace parte del complejo SWItch/sacarosa no fermentable (SWI/SNF), plataforma encargada de la remodelación de la cromatina dependiente de ATP y de otros componentes críticos para el control de la expresión génica, incluyendo la supresión tumoral. Los modelos murinos transfectados de células del AT/RT muestran disminución de la senescencia, aumento de la proliferación y del número de células apoptóticas. La pérdida de expresión del INI-1 causa progresión del ciclo celular, a través de la disminución de la p16 inK4a y aumento de E2Fs y ciclina D1 (figura 5). Sin embargo, el estímulo anormal causado por la pérdida de INI-1 también puede modificar el curso del ciclo celular y la inducción 


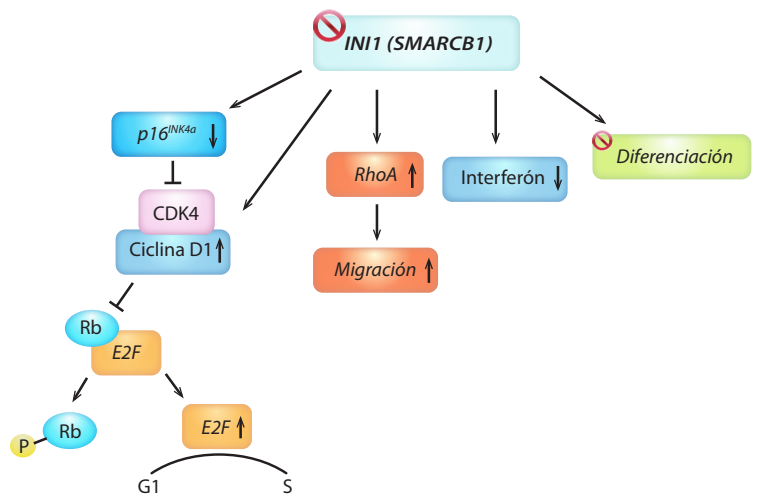

Figura 5. Efectos relacionados con el cáncer secundarios a la pérdida de INI-1 (SMARCB1); el complejo CDK4/ciclina D1 provoca la fosforilación de Rb, lo que conduce a la expresión de gen E2F y a la progresión del ciclo celular. La p16INK4a inhibe la actividad del complejo, el INI-1 regula la señalización de interferón y la ciclina D1 y la p16INK4a podrían constituir objetivos terapéuticos para modular la expresión de la proteína INI-1.

de apoptosis ${ }^{16,17}$. Un estudio reciente describió en varios miembros de una familia con schwannomas y AT/RT una relación hereditaria con la duplicación del exón 6 del INI-1. También mutaciones del gen SMARCA4 en dos hermanas con tumores rabdoides que carecían de alteraciones en la fracción SMARCB1, lo que indicó diversidad germinal en el complejo SWI/SNF para la formación de estas lesiones ${ }^{18}$.

La vía Akt/mTOR, que cumple un importante papel en la regulación del crecimiento celular, en la proliferación, la supervivencia, el metabolismo endógeno, la síntesis proteica y la transcripción, se ha relacionado con el desarrollo del AT/RT. Las células tumorales suelen sobreexpresar receptores de insulina (RI) y para el receptor del factor de crecimiento similar a la insulina tipo 1 (IGF-IR, por su sigla en inglés). Además, las líneas celulares del AT/RT secretan insulina que activa Akt vía expresión de PDX-1 (factor promotor de la insulina tipo 1), un factor de transcripción que regula el desarrollo del páncreas endocrino y modula diversos genes, entre otros, la insulina. En dichos estudios, se detectó una alta expresión de PI3K (fosfatidilinositol 3-quinasa), IR sustrato-1 (IRS-1), mTOR y ERK 1/2. En consecuencia, la manipulación terapéutica de los elementos formes del AT/RT demostró inhibición de la proliferación celular con medicamentos que alteran Akt (perifosine), o con inhibidores de mTOR (sirolimus); no obstante, los inhibidores de ERK1/2 no alteraron el comportamiento del tumor ${ }^{9,19}$.

Lee y colaboradores investigaron el papel de la Aurora Quinasa-A (STK6), un miembro de la superfamilia de las serin/treonin quinasas. La STK6 está relacionada con el reclutamiento de la $\beta$-tubulina y de otras proteínas durante la maduración del centrosoma, el mantenimiento del huso bipolar y la segregación de los cromosomas durante la mitosis ${ }^{20,21}$. Esta quinasa está sobreexpresada en diferentes tipos de cáncer y se ha implicado en los procesos de inestabilidad genómica, en la génesis tumoral y en el pronóstico adverso de diversas neoplasias sólidas; el mismo grupo encontró que las células del AT/RT tienen una elevación significativa de la actividad de esta quinasa, evento que podría modular el curso del ciclo celular, la segregación cromosómica anormal y la apoptosis en este tumor. Con este propósito, se diseñó e inició un experimento clínico fase I (NCT01154816) para probar la dosis biológica efectiva y la toxicidad del MLN 8327 (alisertib) ${ }^{20}$.

La mayor parte del tratamiento del AT/RT en adultos ha sido extrapolada del manejo de los niños; entre ellos se ha encontrado un aumento de la SG en quienes se logra una resección amplia versus subóptima (20 meses y 15.2 meses, respectivamente) ${ }^{8}$. Como parte del manejo sistémico, se han utilizado la temozolamida y el esquema ICE (ifosfamida, carboplatino y etoposido) en conjunto o después de la radioterapia. En estos pacientes (50\% de los casos reportados), la SG oscila entre los 6 meses y los 17 años, con una mediana de 24 meses $^{5}$. La quimioterapia intratecal se ha asociado con una mejor supervivencia en la población de pacientes que no recibió radioterapia ${ }^{8}$. Quienes solo se expusieron a cirugía y teleterapia tuvieron una SG entre 2 meses y 7 años, con una mediana de 9 meses y una tasa de recurrencia local del 75\% después de 1.5 años. Hilden y colaboradores incluyeron 13 pacientes sometidos a quimioterapia primaria seguida de intensificación de dosis más rescate con progenitores extraídos de sangre periférica, intervención con la que se alcanzó una mejoría parcial de los desenlaces más importantes ${ }^{22}$.

La implementación de nuevas intervenciones dirigidas parece una realidad tangible para el AT/RT gracias a la perfilación genética a gran escala que ha permitido desarrollar la línea de los deacetiladores de histonas, de los inhibidores específicos del receptor para el factor de crecimiento similar a la insulina tipo 1 y de los medicamentos que inhiben la Aurora Quinasa-A y la ciclina D1. En Denver (Colorado, EE. UU.), se está explorando la utilidad de la tricostatina $A$, del vorinostat y del entinostat (275-SNDX) ${ }^{23-25}$. Este grupo de medicamentos actúa incrementando la acetilación de las histonas en los residuos de lisina, gracias al bloqueo fisiológico de 


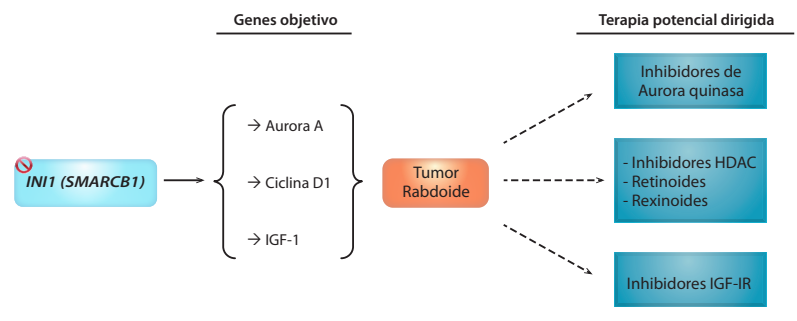

Fuente: adaptado de Ginn KF, Gajjar A. Atypical teratoid rhabdoid tumor: current therapy and future directions. Front Oncol. 2012;2.114.

Figura 6. Posibles intervenciones dirigidas para el control del AT/RT. HDC: histona deacetilasa; IGF-IR: receptor para el factor de crecimiento similar a la insulina tipo 1.

la histona deacetilasa ${ }^{25}$; de igual forma, se ha encontrado una disminución en la capacidad de migración tumoral y en la angiogénesis ${ }^{26}$, así como un aumento potencial en la sensibilidad a la radioterapia ${ }^{27}$. Knipstein y colaboradores demostraron en líneas celulares de AT/RT que el vorinostat a bajas dosis es capaz de disminuir la capacidad de formar colonias y el volumen de estas después de la exposición a la radioterapia ${ }^{23,26}$. Igualmente, los inhibidores mTOR constituyen una herramienta terapéutica promisoria para un sinnúmero de neoplasias neurooncológicas de baja incidencia 28,29,30 (figura 6).

\section{Conclusiones}

Dado lo inusual de los AT/RT en la población adulta, presentamos el octavo caso de localización selar/supraselar discutido en la literatura mundial. Su diagnóstico histológico representó un reto debido a las características morfológicas y a la reactividad diferencial en la inmunohistoquímica, elemento de extrema importancia para caracterizar la pérdida de función del INI-1. En la actualidad, el estudio molecular del AT/RT abarca la evaluación del SMARCB1, así como la vía Akt/mTOR, que podría constituir una opción futura de tratamiento dirigido. En el momento, el abordaje del AT/RT requiere una visión multimodal que incluye una resección quirúrgica máxima, seguida de poliquimioterapia y radioterapia, contemplando en casos seleccionados la intensificación de dosis y el rescate con células progenitores extraídas de médula ósea.

\section{Referencias}

1. Beckwith JB, Palmer NF. Histopathology and prognosis of Wilms tumor: results from the First National Wilms' Tumor Study. Cancer. 1978;41(5):1937-48.

2. Montgomery P, Kuhn JP, Berger PE. Rhabdoid tumor of the kidney: a case report. Urol Radiol. 1985;7(1):42-4

3. Rorke LB, Packer RJ, Biegel JA. Central nervous system atypical teratoid/rhabdoid tumors of infancy and childhood: definition of an entity. J Neurosurgery. 1996;85(1):56-65.

4. Louis DN, Ohgaki $H$, Wiestler OD, Cavenee WK, Burger PC, Jouvet A, et al. The 2007 WHO classification of tumours of the central nervous system. Acta Neuropathol. 2007;114(5):547.

5. Ginn KF, Gajjar A. Atypical teratoid rhabdoid tumor: current therapy and future directions. Front Oncol. 2012;2:114.

6. Samaras V, Stamatelli A, Samaras E, Stergiou I, Konstantopoulou $\mathrm{P}$, Varsos $\mathrm{V}$, et al. Atypical teratoid/rhabdoid tumor of the central nervous system in an 18-year-old patient. Clinical Neuropathol. 2009;28(1):1-10.

7. Packer RJ, Biegel JA, Blaney S, Finlay J, Geyer JR, Heideman $R$, et al. Atypical teratoid/rhabdoid tumor of the central nervous system: report on workshop. J Pediatr Hematol Oncol. 2002;24(5):337-42

8. Shonka NA, Armstrong TS, Prabhu SS, Childress A, Choi S, Langford LA, et al. Atypical teratoid/rhabdoid tumors in adults: a case report and treatment-focused review. J Clin Med Res. 2011;3(2):85-92.

9. Bikowska B, Grajkowska W, Jó囚wiak J. Atypical teratoid/rhabdoid tumor: short clinical description and insight into possible mechanism of the disease. Eur J Neurol. 2011;18(6):813-8.
10. Raisanen J, Biegel JA, Hatanpaa KJ, Judkins A, White CL, Perry A. Chromosome 22q deletions in atypical teratoid/rhabdoid tumors in adults. Brain Pathol. 2005;15(1):23-8.

11. Arita K, Sugiyama K, Sano $T$, Oka H. Atypical teratoid/rhabdoid tumour in sella turcica in an adult. Acta Neurochir (Wien). 2008;150(5):491-6.

12. Las Heras F, Pritzker KP. Adult variant of atypical teratoid/rhabdoid tumor: immunohistochemical and ultrastructural confirmation of a rare tumor in the sella tursica. Pathol Res Pract. 2010;206(11):788-91.

13. Schneiderhan TM, Beseoglu K, Bergmann M, Neubauer U, Macht $\mathrm{S}$, Hänggi $\mathrm{D}$, et al. Sellar atypical teratoid/rhabdoid tumours in adults. Neuropathol Appl Neurobiol. 2011;37(3):326-9.

14. Kuge A, Kayama T, Tsuchiya D, Kawakami K, Saito S, Nakazato $Y$, et al. Suprasellar primary malignant rhabdoid tumor in an adult: a case report. No Shinkei Geka. 2000;28(4):351-8.

15. Meyers SP, Khademian ZP, Biegel JA, Chuang SH, Korones DN, Zimmerman RA. Primary intracranial atypical teratoid/rhabdoid tumors of infancy and childhood: MRI features and patient outcomes. AJNR Am J Neuroradiol. 2006;27(5):962-71.

16. Roberts C, Biegel J. The role of SMARCB1/INI1 in the development of rhabdoid tumors. Cancer Biology \& Therapy. 2009;8(5):412-6.

17. Oruetxebarria I, Venturini $F$, Kekarainen $T$, Houweling A, Zuijderduijn LM, Mohd-Sarip A, et al. P16INK4a is required for hSNF5 chromatin remodeler-induced cellular senescence in malignant rhabdoid tumor cells. J Biol Chem. 2004;279(5):3807-16.

18. Eberhart CG. Molecular diagnostics in embryonal brain tumors Brain Pathol. 2011;21(1):96-104. 
19. Arcaro A, Doepfner KT, Boller D, Guerreiro AS, Shalaby $\mathrm{T}$, Jackson SP, et al. Novel role for insulin as an autocrine growth factor for malignant brain tumour cells. Biochem J. 2007;406(1):57-66.

20. Lee S, Cimica V, Ramachandra N, Zagzag D, Kalpana GV. Aurora $A$ is a repressed effector target of the chromatin remodeling protein INI1/hSNF5 required for rhabdoid tumor cell survival. Cancer Res. 2011;71(9):3225-35.

21. Crane R, Gadea $B$, Littlepage $L, W u H$, Ruderman JV. Aurora $A$, meiosis and mitosis. Biol Cell. 2004;96(3):215-29.

22. Hilden JM, Meerbaum S, Burger P, Finlay J, Janss A, Scheithauer BW, et al. Central nervous system atypical teratoid/rhabdoid tumor: results of therapy in children enrolled in a registry. J Clin Oncol. 2004;22(14):2877-84.

23. Knipstein JA, Birks DK, Donson AM, Alimova I, Foreman NK, Vibhakar R. Histone deacetylase inhibition decreases proliferation and potentiates the effect of ionizing radiation in atypical teratoid/rhabdoid tumor cells. Neuro Oncol. 2012;14(2):175-83.

24. Chi SN, Zimmerman MA, Yao X, Cohen KJ, Burger P, Biegel JA, et al. Intensive multimodality treatment for children with newly diagnosed CNS atypical teratoid rhabdoid tumor. J Clin Oncol. 2009;27(3):385-9.

25. Furchert SE, Lanvers-Kaminsky C, Juürgens H, Jung M, Loidl A, Frühwald MC. Inhibitors of histone deacetylases as potential therapeutic tools for high-risk embryonal tumors of the nervous system of childhood. Int J Cancer. 2007;120(8):1787-94.

26. Xu WS, Parmigiani RB, Marks PA. Histone deacetylase inhibitors: molecular mechanisms of action. Oncogene. 2007;26(37):5541-52.

27. Jung M, Kozikowski A, Dritschilo A. Rational design and development of radiation-sensitizing histone deacetylase inhibitors. Chem Biodivers. 2005;2(11):1452-61.

28. Ronellenfitsch $M$, Steinbach JP, Wick W. Epidermal growth factor receptor and mammalian target of rapamycin as therapeutic targets in malignant glioma: current clinical status and perspectives. Targ Oncol. 2010;5(3):183-91.

29. Prasad G, Sottero T, Yang X, Mueller $S$, James CD, Weiss WA, et al. Inhibition of PI3K/mTOR pathways in glioblastoma and implications for combination therapy with temozolomide. Neuro Oncol. 2011;13(4):384-92.

30. Sukumari-Ramesh S, Singh N, Dhandapani KM, Vender JR. mTOR inhibition reduces cellular proliferation and sensitizes pituitary adenoma cells to ionizing radiation. Surg Neurol Int. 2011;2:22

31. Swensen JJ, Keyser J, Coffin CM, Biegel JA, Viskochil DH, Williams MS. Familial occurrence of schwannomas and malignant rhabdoid tumour associated with a duplication in SMARCB1. J Med Genet. 2009;46(1):68-72. 